\title{
Golden-Rule Level of the Employment
}

\author{
Yong Tao* \\ College of Economics and Management, Southwest University \\ Chongqing, P. R. China \\ taoyingyong@yahoo.com \\ Xiangjun $\mathrm{Wu}$ \\ College of Economics, Hangzhou Dianzi University \\ Hangzhou 310018, P. R. China
}

Received 29 December 2016

Accepted 29 December 2016

Published 26 January 2017

\begin{abstract}
The competitive economy, over a long time scale, would produce a large number of general equilibria, each of which can be regarded as a possible microstate of this economy. Then by the principle of maximum entropy, we can obtain the most probable macrostate which in the case of perfect competition involving a single industry will lead to a Solow-type aggregate production function. By this aggregate production function, one can make clear how labors match firms on the balanced growth path. Here, we prove that when the capital stock of a society arrives at the golden-rule level on the balanced growth path, the social employment will reach the best level at which every firm on average employs an optimal amount of workers.
\end{abstract}

Keywords: Economic growth; golden-rule level; general equilibrium; spontaneous order.

\section{Introduction}

Golden-rule level of capital stock is an important notion in the theory of economic growth $^{1}$. On such a level, a marginal change in saving rate has no effect on consumption in the long run, and consumption is at its maximum possible level among balanced growth paths. In particular, the Phelps-Koopmans theorem asserts that every stationary path with capital stock in excess of the golden rule is inefficient, ${ }^{2}$ as reducing the capital stock can increase the consumption level in all dates. For example, bubbles are generally viewed as a consequence of "dynamic inefficiency" resulting from a capital stock above the golden-rule level ${ }^{3}$; therefore, a natural scheme of eliminating bubbles is to reduce capital stock. Consider that bubbles are

${ }^{*}$ Corresponding author.

This is an Open Access article published by World Scientific Publishing Company. It is distributed under the terms of the Creative Commons Attribution 4.0 (CC-BY) License. Further distribution of this work is permitted, provided the original work is properly cited. 
often regarded as possible triggers of economic crisis; we want to make clear how the action of reducing capital stock will influence the employment level of a society. To arrive at this purpose, we first need to know how labors match firms on the balanced growth path.

Nevertheless, like modern micro-founded macroeconomics, neoclassical growth model is assumed to obey an unduly strong assumption ${ }^{4}$ : Homogeneous agents, each of whom conducts the same behavior. Then the aggregate production function can be simply replaced by the production function of a representative firm (agent). As a result, it is meaningless to discuss how labors match firms, since in such a simplified society there is only one firm. Recently, some pioneering theorists also attempt to introduce heterogeneous behaviors among agents within the framework of the modern micro-founded macroeconomics; however, they soon noted that the Dynamic Stochastic Model will become extremely complex so that the equilibrium solution is hard to find. It is here worth mentioning that physicists in 19th Century also encountered the same dilemma when they attempted to understand the collective behavior of a large number of molecules by solving the Newton equations involving heterogeneous behaviors among many-body. Nonetheless, these physicists finally abandoned solving these excessively intricate Newton equations, and, by an unconventional approach, established the statistical physics.

Statistical physics is an elegant tool for understanding collective behavior of a many-body system. Although the number of firms in an economy is less than the counterpart quantity in physics, there are still $10^{6}$ firms and $10^{7}$ households, and these are large numbers. ${ }^{5}$ Because of this fact, many scholars applied the methods of statistical physics to study the collective behavior of many agents. Indeed, there have been many relevant papers in the literature. ${ }^{5-16}$ Even so, we still have little understanding for the relationship between statistical equilibrium and general equilibria. Recently, Tao ${ }^{12}$ made an effect to clarify the relationship. According to Tao's argument, ${ }^{12}$ the general equilibria actually determine all possible microstates of a given economy, and the statistical equilibrium determines the most probable macrostate which contains the most microstates. Tao ${ }^{17}$ called such a macrostate "Spontaneous Economic Order" (i.e., the most probable economic order). The statistical equilibrium is equivalent to general equilibrium if and only if there is solely a general equilibrium (i.e., a unique microstate). In particular, Tao ${ }^{17}$ showed that if a spontaneous economic order emerges, the aggregate output can be written as a function of firms' number and technological progress, and the empirical tests of Tao's theory were also fully studied by Tao et al. ${ }^{18}$ On the other hand, the neoclassical growth model requires that the aggregate output is generally a function of labor, capital and technological progress. This means that if we combine neoclassical growth model with Tao's spontaneous order model, we may discern how labors match firms in the process of economic growth.

The organization of this paper is as follows. Section 2 shows that a general equilibrium can be identified with a microstate of competitive economy, and the competitive economy, over a long time scale, will produce a large number of general 
equilibria. Section 3 introduces the macrostate of competitive economy, and later, by the principle of maximum entropy, derives the most probable macrostate. Section 4 shows that the most probable macrostate of a perfectly competitive economy involving a single industry would lead to a Solow-type aggregate production function. By this aggregate production function, Sec. 5 investigates the optimal level of employment on the balanced growth path. Section 6 concludes. The main purpose of this paper is to show that, for a perfectly competitive economy, when the capital stock of this economy arrives at the golden-rule level on the balanced growth path, the social employment will reach the best level at which every firm on average employs an optimal amount of workers.

\section{Microstates and General Equilibria}

Following the standard framework of neoclassical microeconomics, ${ }^{19}$ we assume that there are $M$ consumers, $N$ firms and $L$ commodities. Every consumer $i=1, \ldots, M$ is specified by a consumption set $X_{i} \subset R^{L}$, a preference relation $\succsim$ on $X_{i}$, an initial endowment vector $\omega_{i} \in R^{L}$. Each firm $j=1, \ldots, N$ is characterized by a production set $Y_{j} \subset R^{L}$. We denote by $x_{i}=\left(x_{1 i}, \ldots, x_{L i}\right)$ the consumption vector of the $i$ th consumer, where $x_{i} \in X_{i}$ and $x_{k i} \geq 0$ for $k=1, \ldots, L$. We denote by $y_{i}=$ $\left(y_{1 j}, \ldots, y_{L j}\right)$ the production vector of the $j$ th firm, where $y_{j} \in Y_{j}$. Adopting the input-output model's convention, ${ }^{20,21} y_{j}$ 's positive component denotes output and negative component denotes input. Here, we assume that all these firms only produce the $m$-th type of commodity, and specifically we let $y_{m j} \geq 0$ for $j=1, \ldots, N$. The economic meaning of such an assumption is that there is only one industry. ${ }^{12}$ The "industry" in neoclassical economics has a rigid definition: each industry corresponds to a different commodity. Thus, a single industry implies that the society only produces one type of commodity ${ }^{12}$ (e.g., rice). The assumption regarding single output seems very restrictive; therefore, our result works well only for an ideal economy. However, Tao has considered the case of multiple industries. ${ }^{17}$

Given the above economy, if each consumer's preferences are strictly convex and if each firm's production possibility sets are strongly convex, there exists a unique general equilibrium allocation $\left(x_{1}^{c}, \ldots, x_{M}^{c}, y_{1}^{c}, \ldots, y_{N}^{c}\right)$ to guarantee the following three requirements ${ }^{7}$ :

(i) Every consumer's utility is maximal;

(ii) Every firm's profit is maximal;

(iii) Market clearing: $\sum_{i=1}^{M} x_{i}^{c}=\sum_{i=1}^{M} \omega_{i}+\sum_{j=1}^{N} y_{j}^{c}$.

From here we note that a general equilibrium allocation determines a detailed optimal behavior of consumers and firms, and so is the most specific description one can get. ${ }^{12}$ Therefore, the general equilibrium allocation can be regarded as a microstate of the economic system. 
It should be noted that the strong convexity of production possibility sets is not the necessary condition for guaranteeing the existence of general equilibrium. To make matters worse, it will rule out the case of long-run competition. Because of this, we may relax it. In fact, long-run competition is considerably important if we notice that the relaxation time of economic systems is far longer than that of molecular systems. From this claim, we believe that statistical regularities of economic system will emerge over a longer time scale (e.g., one year rather than $10^{-6} \mathrm{~s}$ ) as compared to a molecular system. ${ }^{11}$ Following Marshall's tradition, long-run competition is identified with a belief that every firm can freely enter and exit any industry. ${ }^{19}$ Tao $^{17}$ showed that such a belief leads to two constraints for the production sets:

(iv) (additivity) $Y_{j}+Y_{j} \subset Y_{j}$ for every $j$;

(v) (publicly available technology): $Y_{1}=Y_{2}=\cdots Y_{N}$.

In other words, if a competitive economy satisfies (iv) and (v), it is a long-run competitive economy. It is easy to verify that (iv) and (v) violates strong convexity. Under the aforementioned conditions (i)-(v), Tao ${ }^{17}$ has proved that a long-run competitive economy will have multiple equilibria ${ }^{\mathrm{a}}$

$$
\left(x_{1}^{*}, \ldots, x_{M}^{*}, t_{1} z(p), \ldots, t_{N} z(p)\right)
$$

satisfying (i)-(iii), where $x_{i}^{*}$ for $i=1, \ldots, M$ and $z(p)=\left(z_{1}(p), \ldots, z_{L}(p)\right)$ are fixed vectors, and $\left\{t_{j}\right\}_{j=1}^{N}$ satisfies:

$$
\left\{\begin{array}{l}
t_{j} \geq 0 \quad \text { for } j=1,2, \ldots, N \\
\sum_{j=1}^{N} t_{j}=1
\end{array}\right.
$$

Also, $z(p)$ must obey

$$
p \cdot z(p)=0
$$

where $p=\left(p_{1}, \ldots, p_{L}\right)$ stands for the equilibrium price vector.

Equation (3) guarantees that every firm in the long-run equilibria only obtains zero economic profit. ${ }^{\mathrm{b}}$ Then by Eq. (1), each firm $j$ will obtain $t_{j} p_{m} z_{m}(p)$ units of revenue (or value of output), where $z_{m}(p)$ denotes the $m$ th component of $z(p)$ (namely, output). Since $x_{i}^{*}$ for $i=1, \ldots, M$ is a fixed vector, the equilibrium

a Then $y_{1}^{*}=t_{1} z(p), y_{2}^{*}=t_{2} z(p)$, and so on.

$\mathrm{b}$ Zero economic profit is the inevitable outcome of long-run competition. Since each firm can freely enter and exit the industry, a firm will enter the industry if it can earn positive profits at the going market price and will exit if it can make only negative profits at any positive production level given this price. If all firms, active and potential, take prices as unaffected by their own actions, this implies that active firms must earn exactly zero profits in any long-run competitive equilibrium; otherwise, we would have either no firms willing to be active in the industry (if profits were negative) or an infinite number of firms entering the industry (if profits were positive), see Mas-Collel et al. ${ }^{19}$ 
allocation (1) can be reduced to $\left(t_{1} z(p), \ldots, t_{N} z(p)\right)$. Because each input-output allocation $\left(t_{1} z(p), \ldots, t_{N} z(p)\right)$ satisfying (2) will determine a corresponding revenue allocation, we can replace the input-output allocation $\left(t_{1} z(p), \ldots, t_{N} z(p)\right)$ simply by the revenue allocation $\left(t_{1} p_{m} z_{m}(p), \ldots, t_{N} p_{m} z_{m}(p)\right)$. Furthermore, if we let $\Pi=p_{m} z_{m}(p)$, long-run competitive equilibria $\left(t_{1} p_{m} z_{m}(p), \ldots, t_{N} p_{m} z_{m}(p)\right)$ can be directly written $\operatorname{as}^{17}$ :

$$
\left(R_{1}, R_{2}, \ldots, R_{N}\right)
$$

where $R_{j}$ denotes the revenue of the $j$ th firm and by Eq. (2) satisfies:

$$
\left\{\begin{array}{l}
R_{j} \geq 0 \quad \text { for } j=1,2, \ldots, N \\
\sum_{j=1}^{N} R_{j}=\Pi
\end{array}\right.
$$

In summary, any revenue allocation $\left(R_{1}, R_{2}, \ldots, R_{N}\right)$ satisfying Eq. (5) constitutes a long-run competitive equilibrium. Because a competitive equilibrium represents a microstate, the long-run competitive economy obviously contains a large number of microstates. Next, we will introduce the notion of macrostate.

\section{Macrostate and Spontaneous Economic Order}

The discussion in Sec. 2 indicates that in a long-run competitive economy one can only take into account the behavior of firm. This is because the equilibrium behavior of consumer, $x_{i}^{*}$ for $i=1, \ldots, M$, is fixed: To keep this point, following the assumption highlighted by neoclassical economics ${ }^{17}$ consumer's preference has been assumed to be strictly convex.

The economic implication of Eq. (5) is clear: Every firm will have equal chance of occupying any non-negative revenue level. In fact, here we must impose Rawls' fairness to ensure equal probability, see Ref. 17, but the total revenue of all the firms must equal $\Pi$. Hence, similar to statistical physics, we might use a set of non-negative numbers, $\left\{a_{k}\right\}_{k=1}^{n}=\left\{a_{1}, a_{2}, \ldots, a_{n}\right\}$, to denote a possible macrostate, where each $a_{k}$ represents that there are $a_{k}$ firms, each of which obtains $\varepsilon_{k}$ units of revenue, ${ }^{\mathrm{c}}$ and that these $a_{k}$ firms are distributed among $g_{k}$ industries. Thus, we immediately have the following two constraints:

$$
\begin{aligned}
\sum_{k=1}^{n} a_{k} & =N, \\
\sum_{k=1}^{n} a_{k} \varepsilon_{k} & =\Pi .
\end{aligned}
$$

We are now very interested in counting how many microstates (or general equilibria) a given macrostate will contain. To arrive at this purpose, we next introduce notions of perfect competition and monopolistic competition ${ }^{10,17}$ :

${ }^{\mathrm{c}}$ Here, $0 \leq \varepsilon_{1}<\varepsilon_{2}<\cdots<\varepsilon_{n}$. 
Definition 1. A competitive economy is called a perfectly competitive economy if and only if firms are indistinguishable (or identical).

Definition 2. A competitive economy is called a monopolistic competitive economy if and only if firms are completely distinguishable (or heterogeneous).

Following such two definitions, perfect competition and monopolistic competition obviously obey Bose-Einstein statistics and Boltzmann statistics, respectively. As a result, if one denotes by $\Omega\left(\left\{a_{k}\right\}_{k=1}^{n}\right)$ the number of microstates the macrostate $\left\{a_{k}\right\}_{k=1}^{n}$ contains, then one has ${ }^{17}$ :

$$
\Omega\left(\left\{a_{k}\right\}_{k=1}^{n}\right)= \begin{cases}\prod_{k=1}^{n} \frac{\left(a_{k}+g_{k}-1\right) !}{a_{k} !\left(g_{k}-1\right) !} & \text { (perfect competition) } \\ \frac{N !}{\prod_{k=1}^{n} a_{k} !} \prod_{k=1}^{n} g_{k}^{a_{k}} & \text { (monopolistic competition) }\end{cases}
$$

To distinguish economic terminologies from physical terminologies, $\mathrm{Tao}^{17}$ proposed that the macrostate of an economy could be called "economic order", and that the most probable macrostate could be called "spontaneous economic order". For convenience, we will always call the microstate the equilibrium outcome and call the macrostate the economic order.

It is here worth mentioning that, $g_{k}$ may not be a large number (for example, single industry assumed by us implies $g_{k}=1$ for $\left.k=1,2, \ldots, n\right)$; therefore, we must derive the spontaneous economic order of a perfectly competitive economy $\left\{a_{k}^{*}\right\}_{k=1}^{n}$ carefully.

By the principle of maximum entropy, $\left\{a_{k}^{*}\right\}_{k=1}^{n}$ must maximize $\ln \Omega\left(\left\{a_{k}\right\}_{k=1}^{n}\right)$, which for perfect competition can be written as:

$$
\ln \Omega\left(\left\{a_{k}\right\}_{k=1}^{n}\right)=\sum_{k=1}^{n} \ln \left(a_{k}+g_{k}-1\right) !-\sum_{k=1}^{n} \ln a_{k} !-\sum_{k=1}^{n} \ln \left(g_{k}-1\right) !
$$

Because $a_{k}$ is a large number, by Stirling formula

$$
\ln m ! \approx m(\ln m-1) \quad \text { for } m \gg 1 .
$$

Equation (9) can be rewritten in the form:

$$
\begin{aligned}
\ln \Omega\left(\left\{a_{k}\right\}_{k=1}^{n}\right)= & \sum_{k=1}^{n}\left[\left(a_{k}+g_{k}-1\right) \ln \left(a_{k}+g_{k}-1\right)-a_{k} \ln a_{k}\right. \\
& \left.-g_{k}-\ln \left(g_{k}-1\right) !+1\right] .
\end{aligned}
$$

With the constraints (6) and (7), the Lagrange equation yields:

$$
\frac{\partial \ln \Omega\left(\left\{a_{k}\right\}_{k=1}^{n}\right)}{\partial a_{k}}-\alpha \frac{\partial N}{\partial a_{k}}-\left.\beta \frac{\partial \Pi}{\partial a_{k}}\right|_{a_{k}=a_{k}^{*}}=0
$$

for $k=1,2, \ldots, n$, where $\alpha$ and $\beta$ are Lagrange multipliers. 
Substituting Eq. (10) into Eq. (11) yields:

$$
\ln \left(\frac{a_{k}^{*}+g_{k}-1}{a_{k}^{*}}\right)-\alpha-\beta \varepsilon_{k}=0
$$

for $k=1,2, \ldots, n$.

Thus, we arrive at the spontaneous economic order of a perfectly competitive economy. ${ }^{\mathrm{d}}$

$$
a_{k}=\frac{g_{k}-1}{e^{\alpha+\beta \varepsilon_{k}}-1}
$$

for $k=1,2, \ldots, n$.

By the same method, one easily obtains ${ }^{10,12,17}$ :

$$
a_{k}^{*}(I)=\frac{g_{k}-I}{e^{\alpha+\beta \varepsilon_{k}}-I} \begin{cases}I=1 & \text { (perfect competition) } \\ I=0 & \text { (monopolistic competition) }\end{cases}
$$

for $=1,2, \ldots, n$.

Similar to statistical physics in which the internal energy $U\left(N_{p}, S\right)$ can be written as a function of the number of particles, $N_{p}$ and the entropy $S$, Tao ${ }^{10,17}$ suggested that the total revenue (or gross value of output) of $N$ firms (or agents), $\Pi$, can be written as a function of $N$ and the technological progress $T$; that is,

$$
\Pi=\Pi(N, T) .
$$

By Eqs. (14) and (15), Tao ${ }^{10,17}$ arrived at:

$$
a_{k}^{*}(I)=\frac{g_{k}-I}{e^{\frac{\varepsilon_{k}-\mu}{\lambda \theta}}-I} \begin{cases}I=1 & \text { (perfect competition) } \\ I=0 & \text { (monopolistic competition) }\end{cases}
$$

for $k=1,2, \ldots, n$, where, $\mu=\frac{\partial \Pi}{\partial N}$ and $\theta=\frac{\partial \Pi}{\partial T}$.

It is worth mentioning that technological progress $T$ has been identified with the entropy of the economic system. This means that the improvement of technological progress of a society is due to the degree of freedom that social members have experienced. ${ }^{10,17}$

Equation (16) clearly predicts the revenue distribution among agents (or firms) in a competitive economy. Hence, we can compare it with the household income (like the revenue of self-employed firm) data. Remarkably, some empirical investigations have confirmed that household income distribution of free economies (e.g., USA and United Kingdom) during stable periods obeys Eq. (16) for $I=0$, see Refs. 7, 22, 23; and obeys Eq. (16) for $I=1$ in the run-up to an economic crisis, see Refs. 8 and 9.

d If $g_{k} \gg 1$, then one has $g_{k}-1 \approx g_{k}$. 


\section{The Model}

Equation (16) shows that the multiplicity of agents' behaviors in a free economy, over a long time scale, will yield a spontaneous economic order. We are in particular interested in exploring how labors match firms in such an economic order. To this end, we first investigate the spontaneous economic order of a perfectly competitive economy, $a_{k}^{*}(I=1)$ for $k=1,2, \ldots, n$. Since we have assumed that there is only one industry ${ }^{\mathrm{e}}$ (see Sec. 2), we have

$$
g_{k}=1
$$

for $k=1,2, \ldots, n$.

Proposition 1 [12]. If Eq. (17) holds, then perfect competition implies that each firm occupies the same revenue level $\mu$; that is,

$$
R_{j}=\mu
$$

for $j=1,2, \ldots, N$.

Proof. Since $a_{k}^{*}(I=1)=\frac{g_{k}-1}{e^{\frac{\varepsilon_{k}-\mu}{\lambda \theta}}-1}$ and $g_{k}=1$ for $k=1,2, \ldots, n$, one will have $a_{k}^{*}(I=$ $1)=0$ for $k=1,2, \ldots, l-1, l+1, \ldots, n$, where $\varepsilon_{l}=\mu$. Then by $\sum_{k=1}^{n} a_{k}^{*}(I=1)=$ $N$, one immediately arrives at:

$$
\left\{\begin{array}{l}
a_{l}^{*}(I=1)=N \\
a_{k}^{*}(I=1)=0 \quad \text { for } k=1,2, \ldots, l-1, l+1, \ldots, n
\end{array}\right.
$$

Substituting Eq. (18) into Eq. (5) yields

$$
\Pi=\mu N .
$$

In neoclassical growth model, production function takes Cobb-Douglas form:

$$
z_{m}(p)=A K^{\alpha} L^{1-\alpha} \quad(0<\alpha<1)
$$

where $L$ and $K$ denote aggregate labors and capitals, respectively.

Comparing Eqs. $(20),(21)$ and $\Pi=p_{m} z_{m}(p)$, one has the following proportional relation:

$$
N \sim \Pi \sim z_{m}(p) \sim K^{\alpha} L^{1-\alpha} .
$$

Equation (22) indicates that $N$ should be a function of $L$ and $K$, and so one immediately has:

$$
N(\lambda K, \lambda L)=\lambda N(K, L)
$$

for all $\lambda \geq 0$.

Then by Eqs. (15) and (20), we have

$$
\Pi \sim T \cdot N(K, L) .
$$

e The perfect competition involving multiple industries may induce economic crises; more details see Ref. 10 . 
Therefore, without loss of generality, the production function can be written in the form:

$$
Y=T \cdot N(K, L)
$$

Equation (24) is the core model of this paper, though it holds only for the perfectly competitive economy involving a single industry. Equation (24) together with Eq. (23) illustrate that all firms are of the same size, and every firm occupies the same value of output; therefore, each firm can be regarded as a representative agent. Thus, we have clarified that the "Assumption of Representative Agent" in modern microfounded macroeconomics only holds in an ideal situation where perfect competition involving a single industry is taken into account. Since real economy almost always involves heterogeneous agents, we must use Eq. (16) for $I=0$ to describe it. Then, the production function should yield Eq. (15) rather than Eq. (24). Despite of this, Eq. (24) may still provide some helpful insights for understanding how labors match firms on the balanced growth path.

\section{Employment Level of a Society}

Equation (23), together with (24), implies that technological progress is Hicksneutral. In the spirit of the Solow growth model, we write Eq. (24) in intensive form:

$$
y=T \cdot n(k)
$$

where $y=\frac{Y}{L}, k=\frac{K}{L}$ and $n(k)=\frac{N(K, L)}{L}=N\left(\frac{K}{L}, 1\right)$.

Here, we need to explain the economic meaning of $n(k)$ : It represents the ratio of firms' number to labors' number. From such a ratio, we know that each firm on average hires about $1 / n(k)$ units of labor. Therefore, $n(k)$ essentially reflects the employment level of a society.

Without doubt, we are more interested in the employment level on the balanced growth path.

Assumption 1. The time variable $t$ enters the production function only through $K$ and $L$.

Such an assumption can guarantee that the path of balanced growth for our model does exist ${ }^{\mathrm{f}}$ if Inada conditions are satisfied. In this case, the evolution process of capital stock per unit of labor obeys the following equation:

$$
\frac{d k(t)}{d t}=s y(k(t))-(l+\delta) \cdot k(t)
$$

where $s$ denotes the saving rate, $l$ is the growth rate of labor, and $\delta$ is the depreciation rate of capital.

\footnotetext{
${ }^{\mathrm{f}}$ Because the production function (24) is Hicks-neutral, the corresponding path of balanced growth might not exist if the technological progress factor $T$ is a function of time variable $t$; see Refs. 24 and 25 .
} 


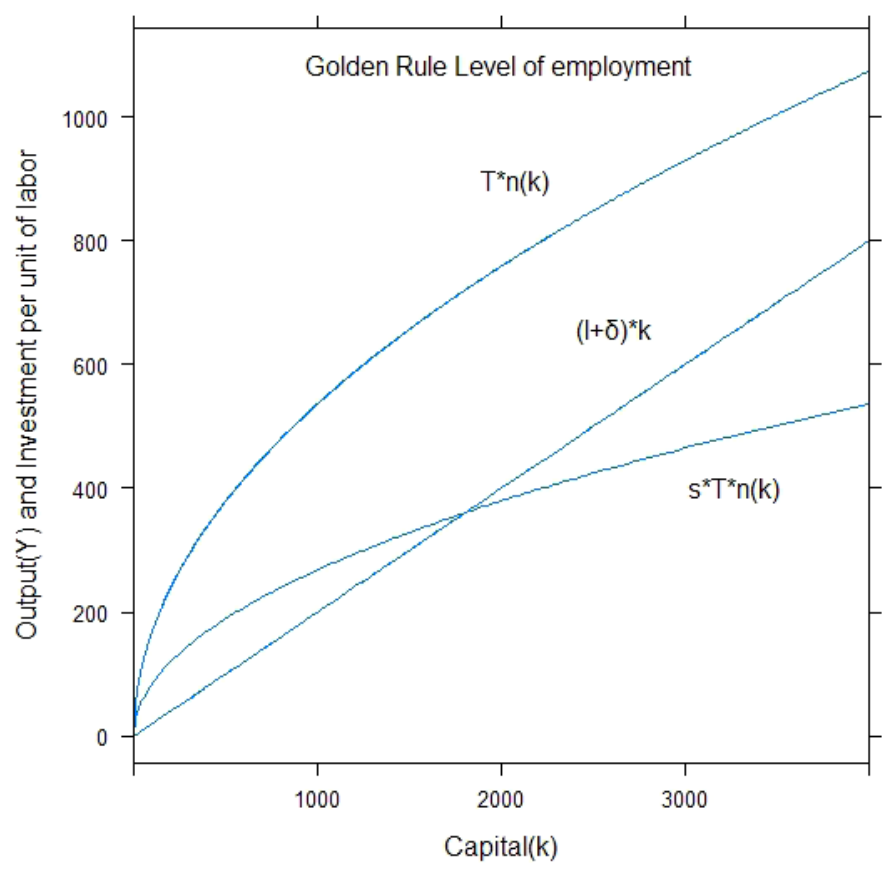

Fig. 1. The capital stock $k^{*}$ on the balanced growth path.

Using Eqs. (25) and (26), the capital stock $k^{*}$ on the balanced growth path is determined by the following equation:

$$
n\left(k^{*}\right)=\frac{l+\delta}{s \cdot T} \cdot k^{*}
$$

The balanced growth path is shown in Fig. 1.

For all balanced growth paths, we are in particular interested in the golden-rule level of capital stock $k_{\mathrm{GR}}$, which will guarantee that

$$
\left.\frac{d y(k)}{d k}\right|_{k=k_{\mathrm{GR}}}=(l+\delta)
$$

or equivalently,

$$
\left.\frac{d n(k)}{d k}\right|_{k=k_{\mathrm{GR}}}=\frac{(l+\delta)}{T}
$$

As such, when each firm on average hires about $1 / n\left(k_{\mathrm{GR}}\right)$ units of labor, a marginal change in $s$ has no effect on consumption in the long run, and consumption is at its maximum possible level among balanced growth paths. Correspondingly, we can call $n\left(k_{\mathrm{GR}}\right)$ the golden-rule level of employment. Now, we attempt to check the bubble economy with capital stock in excess of the golden rule level, i.e., $k^{*}>k_{G R}$. In this case, reducing capital stock, according to Eq. (27), will guarantee that $n\left(k^{*}\right)$ is 
falling, and $1 / n\left(k^{*}\right)$ is therefore rising. This means that the action of reducing capital stock itself in the case of bubble economy not only increases the consumption level but also improves employment. Of course, our argument is based on a closed economy; for the case of open economies, ${ }^{11}$ we need further discussion.

\section{Conclusion}

In the spirit of neoclassical microeconomics, a general equilibrium ensures an optimal behavior of consumer and firm, so that each consumer's utility and each firm's profit are maximal, respectively. Also, this is the most specific description for economic agent's optimal behavior. Therefore, a general equilibrium can be identified with a microstate of the economic system. Tao ${ }^{12,17}$ showed that a competitive economy, over a long time scale, would produce a large number of general equilibria. Then by the principle of maximum entropy, we immediately obtain the most probable macrostate that contains the most equilibrium outcomes. We call such a macrostate the "Spontaneous Economic Order". In particular, the spontaneous order in the case of perfect competition involving a single industry will lead to a Solow-type aggregate production function that is characterized in terms of the number of firms. By this production function, we have investigated how labors match firms on the balanced growth path.

As is well known, the Phelps-Koopmans theorem asserts that every stationary path with capital stock in excess of the golden rule is inefficient. Naturally, a possible scheme of improving efficiency is to reduce capital stock. Our investigation shows that such a scheme not only increases the consumption level of a society but also improves employment. Thus, our investigation approves the feasibility of this scheme. In particular, when capital stock reaches the golden-rule level, every firm will on average match an optimal amount of labors. This case can be viewed as the golden-rule level of employment.

However, it is important to realize that the above procedure would establish the optimal level of employment if and only if the perfect competition involving a single industry is taken into account. Therefore, we cannot answer whether such an optimal level of employment will be established in a monopolistic competitive economy. To address this question, we need to make clear how heterogeneous firms match labors. We wish to complete this task in the future work.

\section{Acknowledgments}

This work was supported by the Fundamental Research Funds for the Central Universities (Grant No. SWU1409444).

\section{References}

1. D. Romer, Advanced Macroeconomics 2nd edn. (McGraw-Hill Companies, 2000).

2. T. Mitra and D. Ray, On the Phelps-Koopmans theorem, J. Econ. Theory 147 (2012) 833-849. 
3. D. Acemoglu, Introduction to economic growth, J. Econ. Theory 147 (2012) 545-550.

4. M. Aoki and H. Yoshikawa, Non-self-averaging in macroeconomic models: A criticism of modern micro-founded macroeconomics, J. Econ. Interact. Coord. 7 (2012) 1-22.

5. H. Aoyama, H. Yoshikawa, H. Iyetomi and Y. Fujiwara, Productivity dispersion: Facts, theory, and implications, J. Econ. Interact. Coord. 5 (2010) 27-54.

6. D. A. Foley, statistical equilibrium theory of markets, J. Econ. Theory 62 (1994) 321-345.

7. V. M. Yakovenko and J. B. Rosser, Statistical mechanics of money, wealth, and income, R. Mod. Phys. 81 (2009) 1703-1725.

8. F. V. Kusmartsev, Statistical mechanics of economics I, Phys. Lett. A 375 (2011) 966.

9. K. E. Kürten, and F. V. Kusmartsev, Bose-Einstein distribution of money in a free-market economy. II, Europhys. Lett. 93 (2011) 28003.

10. Y. Tao, Competitive market for multiple firms and economic crisis, Phys. Rev. E 82 (2010) 036118.

11. Y. Tao and X. Chen, Statistical physics of economic systems: A survey for open economies, Chin. Phys. Lett. 29 (2012) 058901.

12. Y. Tao, Universal laws of human society's income distribution, Physica A 435 (2015) $89-94$.

13. O. Flomenbom, The society-deciders model and fairness in nations, Biophys. Rev. Lett. $10(2015) 157$.

14. Y. Adiguzel, O. Flomenbom and C. Gul, From the Physiocrats to Fairness in Nations, Rep. Adv. Phys. Sci. Special Issue on Socio Econophysics (2017), forthcoming.

15. R. Lopez-Ruiz et al., About Gas-like Economic Models, Repo. Adv. Phys. Sci. Special Issue on Socio Econophysics (2017), forthcoming.

16. O. Flomenbom et al., The Gini of Fairness, Rep. Adv. Phys. Sci. Special Issue on Socio Econophysics (2017), forthcoming.

17. Y. Tao, Spontaneous economic order, J. Evol. Econ. 26 (2016) 467-500.

18. Y. Tao, X. Wu, T. Zhou, W. Yan, Y. Huang, H. Yu, B. Mondal and V. M. Yakovenko, Universal exponential structure of income inequality: Evidence from 60 countries, arXiv: http://arxiv.org/pdf/1612.01624.

19. A. Mas-Collel, M. D. Whinston and J. R. Green, Microeconomic Theory (Oxford University Press, 1995).

20. H. R. Varian, Microeconomic Analysis, 3rd edn. (Norton \& Company, New York, 1992).

21. G. A. Jehle and P. J. Reny, Advanced Microeconomic Theory 2nd edn. (AddisonWesley, 2001).

22. Y. Tao, An Index Measuring the Deviation of a Real Economy from General Equilibrium: Evidence from the OECD Countries, Available at SSRN (2016).

23. F. Clementi, M. Gallegati and G. Kaniadakis, A new model of income distribution: The $\kappa$-generalized distribution, J. Econ. 105 (2012) 63-91.

24. E. S. Phelps, Second essay on the golden rule of accumulation, Amer. Econ. Rev. 55 (1965) 793-814.

25. E. Burmeister and A. R. Dobell, Mathematical Theories of Economic Growth (Macmillan, 1970, New York). 